\title{
The Influence of Culture on ABMP Negotiation Parameters
}

\section{Gert Jan Hofstede Catholijn M. Jonker Tim Verwaart}
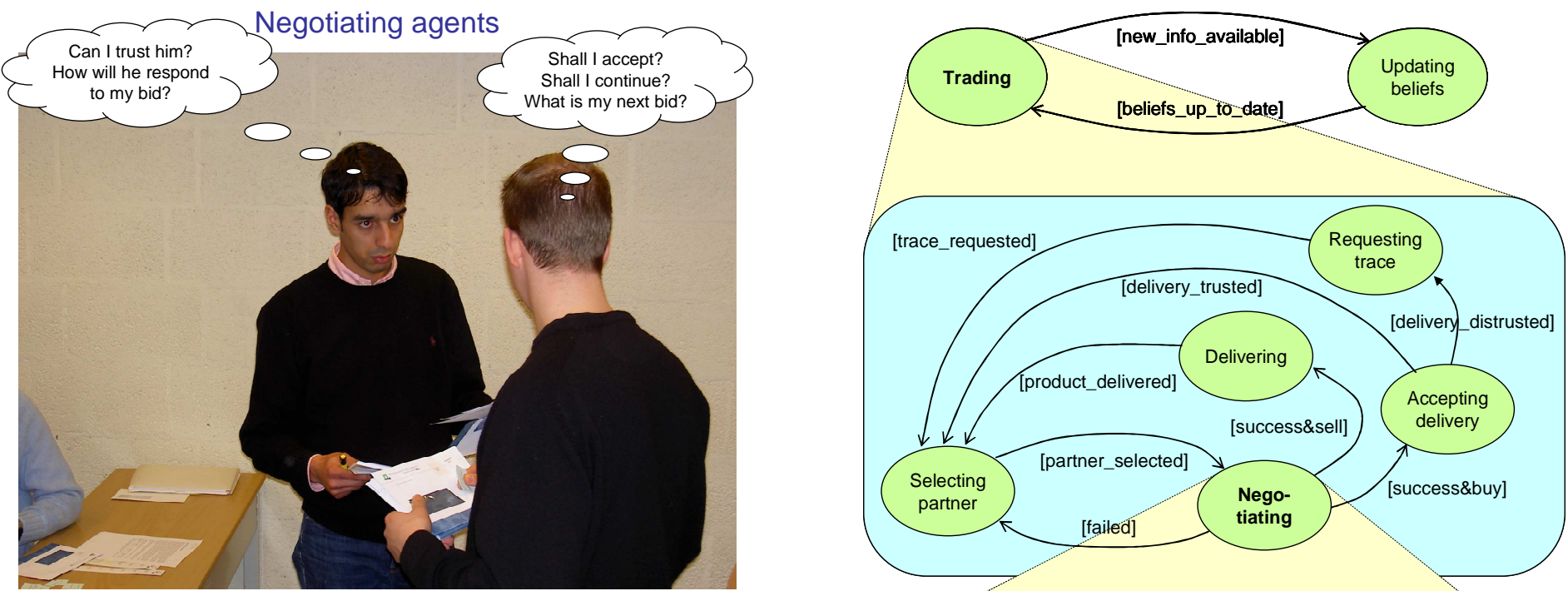

Agent-Based Market Protocol negotiation architecture: [Jonker and Treur, 1999]

Based on comparing utility of bids and parameters for decision rules.

In this case the utility function is a linear combination of economic value, quality preference, and risk evaluation:

$$
U=w_{V} V+w_{Q} Q+w_{R} R
$$

Decision rule parameters:

- concession factor $\gamma$.

how far is the agent willing to go in making concessions?

- negotiation speed $\beta$ :

the extent of concessions the agent typically makes per negotiation round - acceptable utility gap size $\omega$ :

at what utility difference does the agent accept a partner's bid?

- impatience factor $l$ :

inclination to break-off if partner's bids are too far away from the expected

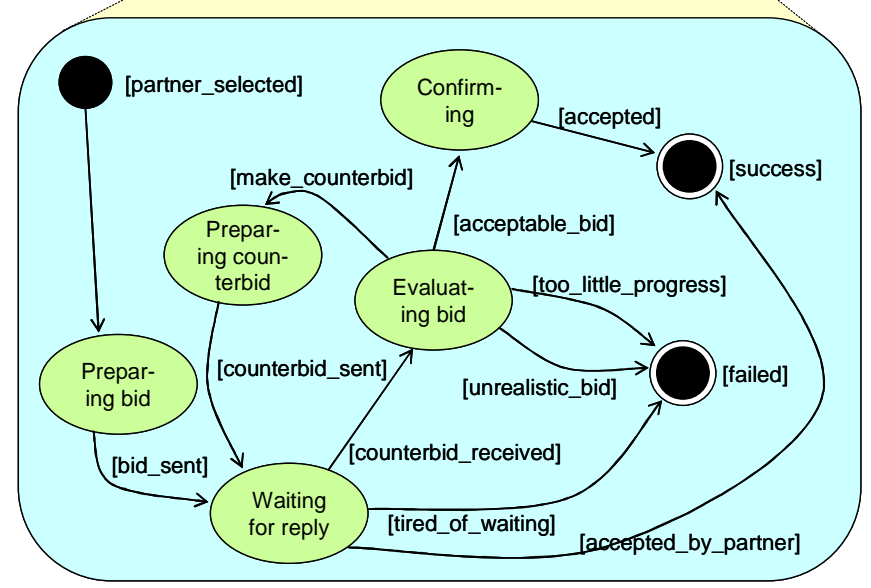

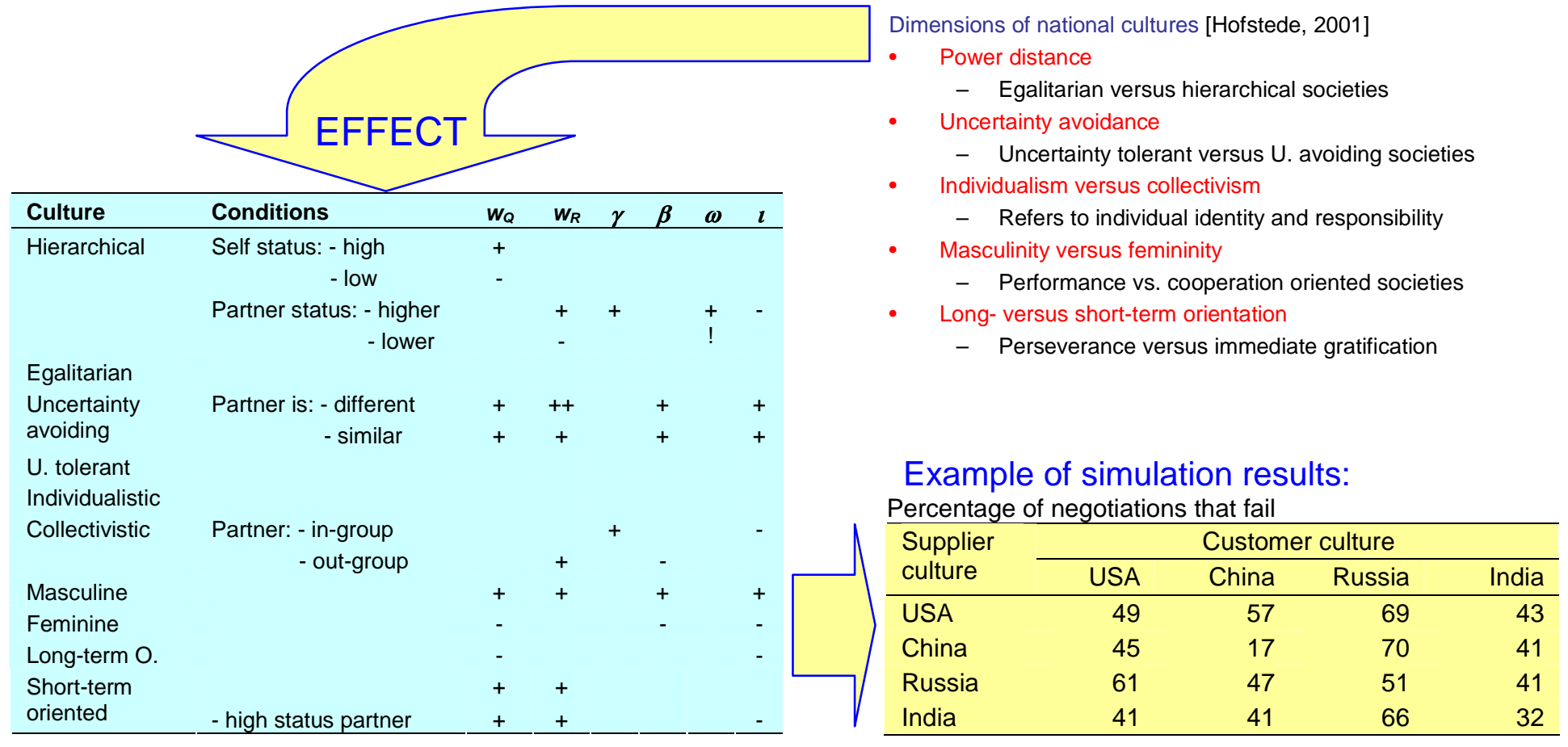




\title{
The Influence of Culture on ABMP Negotiation Parameters: Extended Abstract ${ }^{1}$
}

\author{
Gert Jan Hofstede $^{\mathrm{a}, \mathrm{b}} \quad$ Catholijn M. Jonker $^{\mathrm{b}}$ Tim Verwaart $^{\mathrm{c}}$ \\ ${ }^{a}$ Wageningen University, Hollandseweg 1, 6706 KN Wageningen \\ ${ }^{b}$ Delft University of Technology, Mekelweg 4, 2628 CD Delft \\ ${ }^{c}$ LEI Wageningen UR, Alexanderveld 5, 2585 DB Den Haag
}

\section{Introduction}

Anybody with experience in international trade knows that bargaining practices differ across the world. Models to describe bargaining, are not valid across the world unless culture is taken into account. 'Culture' is a notion with many meanings, some of which are contested in some disciplines. However, the leading paradigm today is widely accepted and used in both practice and academia. According to it, culture refers to the unwritten rules of society. An agent-based model of bargaining in which the agents are cultured offers several promises. It can help understand the dynamics of international negotiations in trade. It could also serve as a training tool for aspiring international traders.

This paper describes an agent-based model for bargaining in the context of trade. The agents follow common sense strategies such as maximizing gain, seeking good quality, and minimizing risk. But they also have models of how to behave in an appropriate manner, such as: is it allowable to refuse a negotiation proposal; may an agent quit if it no longer likes the negotiation; must a serious concession be shown in each bid; is cheating allowed if the partner is not paying attention? These models are based on Hofstede's five dimensions of culture (see [2] for an explanation of these dimensions).

For the agents' negotiation strategy we chose the ABMP architecture of Jonker and Treur [3]. It is based on comparing the utility of bids. The present paper applies the utility function proposed by Tykhonov et al. [4]. It covers the relevant aspects mentioned above (business value, quality and risk):

$$
U(b, a, p)=w_{P, a p} P(b, a, p)+w_{Q, a p} Q(b, a)+w_{R, a p} R(b, a, p)
$$

$U(b, a, p)$ stands for the utility that agent $a$ expects from bid $b$ made by agent $p . P(b, a, p)$ reflects $a$ 's belief about the economic value of the transaction. It is calculated as the profit expected from the transaction in case of cooperation, minus the estimated risk of the transaction. $Q(b, a)$ reflects the subjective valuation of the quality attribute of the proposed transaction, in addition to the market value, e.g. a trader may prefer trading biologically grown food, even if more profit may be made with traditionally grown. $R(b, a, p)$ reflects $a$ 's subjective, risk-averse valuation (in addition to the "rational" risk evaluation included in $P$ ).

The ABMP strategy has a number of parameters, with which the behavior of the agent can be tuned. With respect to the influence of culture, the relevant ABMP parameters are:

- concession factor (how far is the agent is willing to go in making concessions?),

- negotiation speed (the extent of concessions the agent typically makes per negotiation round),

- acceptable utility gap size (at what utility difference does the agent accept a partner's bid?), and - impatience factor (the inclination to break-off if the partner's bids are too far away from the expected).

[1] explains how Hofstede's culture dimensions influence the weight factors in the utility function and the relevant ABMP parameters (increase or decrease according to scores on Hofstede's dimensions whish are given as agent parameters). Furthermore, [1] proposes formal models for the implementation of culturally differentiated ABMP negotiation in agents.

\footnotetext{
${ }^{1}$ Extended abstract of a paper originally presented at ACAN 2009, Budapest, 12 May 2009 [1]: http://www.verwaart.nl/culture/ACAN2009CultureABMPNegotiation.pdf
} 


\section{Simulation Results}

Negotiations are performed in a multi-agent simulation. Eight agents are assigned the role of supplier, eight agents the role of customer. Agents may select a partner and negotiate purchasing of a commodity that has either high or basic quality. However, quality is not visible, so the buyer of a high quality product has to accept risk, i.e. trust the seller. In the current simulation, agents have no information about partner's trustworthiness. If they agree on high quality, they implicitly accept the risk of deceit. Transactions may fail if concession factors cannot bridge the acceptable utility gap, or if impatient agents find progress too slow. Macro level observables of the simulation are number of successful transactions, failed negotiations, average duration of negotiations, average quality of transactions, etcetera.

The correct implementation of Hofstede's model is verified in simulations with agents from imaginary cultures that differ on only one of the dimensions. Tables 1 and 2 present example results of more realistic, complex cultures. A high number of successful transactions (table 1) indicates a rapid flow of trade. Failure of negotiations (table 2) is one of the possible causes that slow trade down. The examples illustrate that the culture parameters have their effect in the multi-agent simulations. They differentiate aggregate performance in mono-cultural settings as well as intercultural interactions in a believable way.

Table 1. Number of successful transactions per run in 16 simulation runs of equal length, each run with different cultural settings

\begin{tabular}{lrrrr}
\hline \multirow{2}{*}{$\begin{array}{l}\text { supplier } \\
\text { culture }\end{array}$} & \multicolumn{4}{c}{ customer culture } \\
\cline { 2 - 5 } & USA & China & Russia & India \\
\hline USA & 61 & 45 & 37 & 69 \\
China & 65 & 90 & 37 & 53 \\
Russia & 49 & 56 & 59 & 63 \\
India & 58 & 61 & 39 & 69 \\
\hline
\end{tabular}

Table 2. Percentage of negotiations that failed per run in 16 simulation runs of equal length, each run with different cultural settings

\begin{tabular}{lrrrr}
\hline \multirow{2}{*}{$\begin{array}{l}\text { supplier } \\
\text { culture }\end{array}$} & \multicolumn{4}{c}{ customer culture } \\
\cline { 2 - 5 } & USA & China & Russia & India \\
\hline USA & 49 & 57 & 69 & 43 \\
China & 45 & 17 & 70 & 41 \\
Russia & 61 & 47 & 51 & 41 \\
India & 41 & 41 & 66 & 32 \\
\hline
\end{tabular}

\section{Conclusion}

Culturally differentiated negotiating agents are useful in a context where human factors play a role. Social simulation is an example of such a context. Other application areas may be training and education, and decision support systems for human negotiations. Cultural differentiation of behavior is less relevant in situations where the purpose of negotiating agents is to outperform people by rational decision making.

This paper contributes to the understanding of culture's influence on decision making in business by exploring the feasibility of Hofstede's five-dimensional model to simulate believable agents in business. Preliminary results of the simulation of complex, reality-based cultures give evidence that culture in agents can be simulated plausibly by applying Hofstede's model. However, more validation is required (on the basis of culture and negotiation literature and experiments) and remains for future research.

\section{References}

[1] G.J. Hofstede, C.M. Jonker, T. Verwaart. The Influence of Culture on ABMP Negotiation Parameters. ACAN 2009 The Second International Workshop on Agent-based Complex Automated Negotiations, Budapest, 10-15 May 2009.

[2] G. Hofstede. Culture's Consequences, Second Edition. Sage Publications, 2001.

[3] C.M. Jonker, J. Treur. An agent architecture for multi-attribute negotiation. In: B. Nebel, Ed. Proceedings of IJCAI '01, 1195-1201. Morgan Kaufman, 2001.

[4] D. Tykhonov, C. Jonker, S. Meijer, T. Verwaart. Agent-Based Simulation of the Trust and Tracing Game for Supply Chains and Networks. J. of Artificial Societies and Social Simulation 11, 2008. 